\title{
Tangence
}

\section{Trente années de Kaosmos : sept arguments sur l'Odin Teatret}

\section{Nicola Savarese}

Numéro 46, décembre 1994

Un théâtre de passage

URI : https://id.erudit.org/iderudit/025840ar

DOI : https://doi.org/10.7202/025840ar

Aller au sommaire du numéro

Éditeur(s)

Tangence

ISSN

0226-9554 (imprimé)

1710-0305 (numérique)

Découvrir la revue

Citer cet article

Savarese, N. (1994). Trente années de Kaosmos : sept arguments sur l'Odin

Teatret. Tangence, (46), 54-71. https://doi.org/10.7202/025840ar d'utilisation que vous pouvez consulter en ligne.

https://apropos.erudit.org/fr/usagers/politique-dutilisation/ 


\section{Trente années de Kaosmos: sept arguments sur l'Odin Teatret Nicola Savarese}

\section{Illusions perdues}

Au fil du temps je me suis fait une conviction: je crois que le théâtre est un phénomène lié à la jeunesse, non pas à la jeunesse idéale (qui déclarerait jamais se sentir vieux?) mais à la jeunesse réelle, celle de l'état civil: passé un certain âge, on ne croit plus au théâtre, ou mieux, on perd l'illusion et on entre dans l'âge du désenchantement.

Quand je dis "on perd l'illusion", je n'entends pas le détachement des rêves et des belles espérances que le jeune âge plus que les autres alimente, mais j'entends la perte de l'illusion théâtrale c'est-à-dire le fait de croire à la fiction propre du théâtre, à laquelle, en tant que jeunes spectateurs, nous n'opposions que peu ou pas du tout de résistance. Du reste, ne pas céder aux illusions veut dire exactement ceci: ne plus croire au jeu. On n'est plus illusionné parce qu'on n'est plus, littéralement, dans le jeu ("in ludum", n'est-ce pas?'1).

Ne plus croire au théâtre ne veut pas dire toutefois perdre la passion du thêâtre. Pour être plus clair, ne plus croire au thêâtre tout en conservant la passion, pour moi cela voulait dire "ne plus croire aux spectacles", c'est-à-dire à la majeure partie des représentations qui se font, et être, en revanche, toujours plus fasciné par le "théâtre d'acteur" qui est véritablement un spectacle rare.

\section{Objections}

Je suis d'accord avec la première objection: tout ce qui survient avec le passage du temps pourrait s'appeler l'affinement progressif du goût. En admettant que le goût puisse se raffiner

1 En français dans le texte. Le présent texte est une traduction. 
avec l'âge (je pense qu'il devient simplement plus subjectif), je pourrais être d'accord seulement si l'on accorde au mot "goût" le même sens et la même valeur que les Indiens confèrent au terme rasa: en sanskrit, rasa est la saveur, la saveur profonde, celle qui - qu'elle soit prononcée ou délicate - est efficace, parce qu'elle reste dans la tête plus que dans le palais et parce que, à l'extérieur comme à l'intérieur de la métaphore, elle est suc et essence: l'essence provocatrice de la poésie est de cette sorte. Du reste, sans aller aussi loin, même chez nous "saveur" vient de "Savoir" et en partage la racine et les risques.

Je suis moins d'accord avec la seconde objection: comment fait-on pour ne pas croire aux spectacles tout en croyant au thêâtre d'acteur? Que l'on excuse pour l'instant la répétition du verbe croire mais il n'existe pas de synonymes aussi convaincants: en s'adressant aux acteurs, Stanislavski avait l'habitude de dire "je te crois " ou bien "je ne te crois pas" sans crainte d'être mal compris. Donc: à la majeure partie des spectacles je ne crois pas, parce que leurs acteurs sont peu crédibles. Il existe par contre de rares spectacles où le problème ne se pose pas et où je n'ai pas l'ombre d'un doute: je suis en face d'un théâtre d'acteur.

Donc ce qui m'arrive, qui m'est déjà arrivé, c'est que j'éprouve de l'impatience tant vis-à-vis des spectacles dans lesquels les acteurs font de leur mieux pour être "naturels", que vis-à-vis de ceux où, à l'inverse, les acteurs "veulent s'exprimer" à tout prix. J'éprouve plus d'impatience encore à l'égard des acteurs qui, pour combattre le naturalisme traditionnel ainsi que toute forme de convention théâtrale, déprécient la nature humaine, avant même celle de l'acteur, en se comportant en scène de manière totalement "contre nature". Au bout du compte je ne pense pas être le seul à voir au théâtre des acteurs ennuyeux qui récitent avec précipitation et mauvaise grâce comme à la télévision (il s'agit en effet très souvent des mêmes): et je ne pense pas être le seul à avoir éprouvé de l'embarras, si ce n'est de la honte, face à des acteurs qui, pour refuser le langage de la télévision ou du théâtre commercial, tombent dans un excès d'expressivité et de fureur qui porte leur jeu à des niveaux insupportables.

On dira que la faute est imputable aux metteurs en scène: cela se peut et c'est même souvent le cas. Mais je pense que c'est aussi la faute des écoles, des académies et des universités où l'on enseigne le théâtre. Pour améliorer son produit, on a recours à 
des procédures sommaires et à des méthodes plus appropriées à la préparation d'un cocktail qu'à celle d'un acteur: un peu de Stanislavski et un peu de Brecht, tant de commedia dell'arte et une pincée de Grotowski...

Mais je ne veux pas discuter ici de modes, d'écoles, ou d'épigones expéditifs. À mon âge (ah! ah!) ce qui m'intéresse c'est l'incrédulité : je n'y crois pas et c'est tout.

\section{Les règles du jeu}

Il existe en revanche des théâtres d'acteur dans lesquels le problème de croire, comme je le disais, ne se pose même pas. C'est ainsi que je m'entête dans ma conviction: la jeunesse passée, à l'âge du désenchantement (ce qui est au fond tout ce qui reste), je suis profondément attiré non pas par l'histoire, mais par la façon de la raconter, non par le jeu mais par les règles du jeu. Donc, pas la fiction théâtrale mais ses conventions, et par conséquent, les acteurs qui privilégient un jeu technique, suivant des règles établies. Peu importe si ce jeu semble légitimé par de nombreuses normes, s'il apparaît codifié et s'il tient de codes qui sont le fruit des siècles, de l'expérience ou d'autres cultures - la danse classique occidentale, le kabuki japonais... - ou qui sont le résultat d'une invention moderne, ou d'une seule pensée théâtrale, comme dans le cas du mime de Decroux: ce qui importe, c'est que les théâtres d'art — par l'entremise de l'acteur — fassent appel de manière contemporaine à mon sens esthétique, à mon intelligence, à mon plaisir. En un mot, à mon credo.

En somme: je crois à la technique, à la rigueur et à la complexité. Je crois à un acteur versatile, capable de moduler le corps et la voix de façon à chanter et à danser, non pas tour à tour, mais de façon à ce qu'en parlant il chante et qu'en se déplaçant il semble danser. Je crois aux acteurs non émotionnés mais capables de susciter des émotions. Je crois aux acteurs qui ne décrivent pas la réalité mais y font allusion en la suggérant. Et je crois à un spectacle où aucun détail n'est laissé au hasard et où tout se laisse découvrir dans la lucidité, aux règles de l'art à travers l'artifice et l'âme, pour dire comme les anciens, l'unité et la simultanéité, pour dire comme les modernes.

Et enfin je crois à cet acteur capable de faire oublier que la science du théâtre existe, selon cette interprétation surprenante 
que l'historien du théâtre Ferdinando Taviani a donné du proverbe ancien "Apprends l'art et mets-le de côté": ce qui ne veut pas dire "n'oublie pas un art appris qui tôt ou tard te servira" mais plutôt "une fois apprise la technique, mets-la en pratique et fais en sorte qu'elle ne prévale pas.

C'est pourquoi je suis attiré, même transporté, par les grandes traditions théâtrales qui professent des règles particulières et qui forment des acteurs à des niveaux si élevés qu'on peut en mesurer chez chacun l'excellence. Ainsi je me réfugie dans les grandes traditions de l'Asie - le Kabuki, le Nô, l'opéra de Pékin, les thêâtres dansés de l'Inde ou de Bali : épurées et enrichies par des siècles d'expérience, ces grandes traditions se meuvent entre virtuosité et naïveté, hardiesse et subtilité, sur le fil d'une réalité hyperréelle. Parce qu'au fond, le point crucial est toujours le même: le thêâtre ne doit pas recréer la réalité mais en dévoiler le double.

Ainsi, quand en Occident je veux voir un thêâtre d'art, un théâtre d'acteur qui me surprenne et me divertisse, qui déjoue mes attentes et me fasse réfléchir, je vais voir un spectacle de l'Odin Teatret. Une fois tous les deux ans environ. Le dernier spectacle que j'ai vu a été Kaosmos. Le rituel de la porte.

\section{Le flux des impressions}

Quand l'Odin joue avec les choses, il le fait avec économie: si, comme dans ce spectacle, une porte est prévue, soyez sûrs que la porte y sera (sain réalisme), qu'elle n'aura pas été achetée en vain (saine économie), qu'elle sera exploitée de toutes les manières possibles et imaginables, y compris comme une porte (fantaisie sensée et insensée).

De combien de façons peut-on frapper à une porte? Si vous voulez les connaître toutes plus une, demandez-le à Iben Nagel Rasmussen elle-même: des coups qu'elle donne, elle n'en donne jamais deux pareils, et pourtant c'est toujours elle. Quand elle frappe à la porte elle ne fait pas autre chose: elle frappe seulement à une porte, mais dans ces coups-là il semble que soient concentrées mille manières de frapper. La manière est: je voudrais entrer, puis-je? Le sens est: c'est toujours moi, tu te souviens? Et dessus, dessous, tout autour on lit: je ne crois pas que ce sera la dernière, mais combien de temps durera-t-elle? Et on 


$$
\text { 圆 }
$$


se demande: de quoi est-elle en train de parler, de la porte ou de la guerre? Et on n'a pas le temps parce que, maintenant, la porte est devenue un lit de mort pour l'homme-qui-ne-veut-pas-mourir mis en croix : est-ce un pauvre christ ou est-ce vraiment le Christ? Et le Christ dirait: quelle différence cela fait-il? Et ceci aussi est juste et bon. Comme elle est amusante cette porte qui devient tantôt cercueil tantôt petite barque, tantôt vaisseau aux voiles déchirées tantôt cette tombe piétinée qui est le monument funéraire du monde.

Il y a une longue scène dans laquelle l'homme-qui-ne-veutpas-mourir - interprété par un stupéfiant Torgeir Wethal - reste immobile, assis sur la porte couchée: c'est une immobilité en marche, oh! si elle marche, elle s'enfuit plutôt comme une spirale ou un terrible $j^{\prime} a c c u s e^{2}$. Ce que dit le franc-tireur à son compagnon: le problème avec les enfants c'est qu'ils ne tiennent pas en place : est-ce seulement l'illustration satyrique d'un journal ou une réalité de Sarajevo? Même chose si l'épouse du village se désespère sur son homme mort, allez donc!; si elle chante à tout le monde son désespoir, allez donc encore; et si la maman ne veut plus reprendre son fils après avoir offert à la mort ses yeux pour le ravoir, allez donc toujours. Bien qu'aveugle, la mère a vu juste: à son fils étaient réservés douleur et misère, horreur et malheur. Après l'holocauste on disait jamais plus, nevermore après le Vietnam et nunca mas après les desaparecidos de l'Amérique latine: attendons-nous, d'un moment à l'autre, aux versions en langue slave et dans les dialectes du Rwanda.

S'il y a une chose qui ne se fait pas c'est de danser sur les tombes et de les piétiner: si vous voulez y manger et y boire, allez-y sans cérémonies, comme au Mexique le deux novembre à la fête de la calavera. Mais danser sur les tombes, surtout pas. Et pourtant quand arrivent tous les acteurs pour le faire, on sent le lourd poids de l'indécision: on ne sait s'il faut crier d'horreur ou de beauté. Ils sont beaux, ces jeunes, comme le claquement de leurs fouets, spécialement quand ils se dépouillent de leurs vieux costumes et les ensevelissent: mais la chanson qu'ils chantent, le piétinement sur la tombe font frissonner.

Comme des autres spectacles de l'Odin, de celui-ci aussi je sors comme étourdi : je reste obsédé par la musique et en particu-

2 En français dans le texte. 


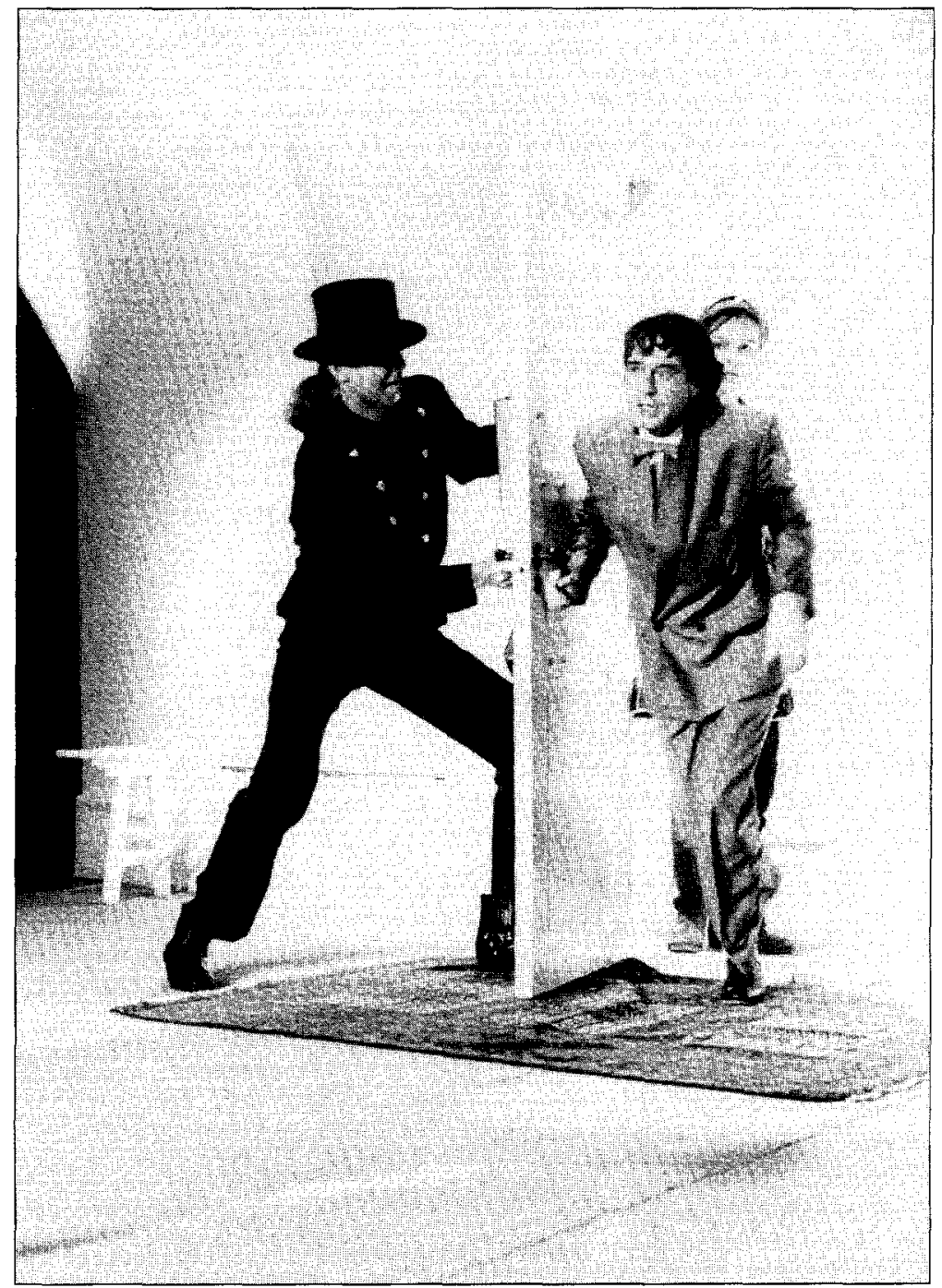

Acteurs: Jan Ferslev, Torgeir Wethal, Tina Neilsen.

(C) Jan Rüsz fotografi 
lier par une mélodie que je ne parviens cependant pas à siffler. Je sais que je perdrai des heures à tenter de me la rappeler. Je me rappellerai de détails même avec la distance, comme si je l'avais vu une minute plus tôt et, en revanche, j'oublierai des scènes entières que je désirerai pourtant pouvoir reconstruire telles quelles. Je me perds en route comme dans les films de Fellini.

Toutefois le spectacle m'apparaît solaire, joyeux. Joyeux? Connaissant Barba, je pense ne pas avoir bien compris les textes que chaque acteur dit dans sa propre langue maternelle. Je feuillette le programme, je lis les pages de Ferenc Gombai, "le vieil érudit hongrois au visage d'écorce d'arbre appuyé au bras de sa septième femme": c'est de celles portant sur le rituel de la porte qu'a été tiré le spectacle. Il me paraît étrange ce vieillard: il parle exactement avec les mêmes mots et les mêmes images que Barba... J'ai un vague soupçon: qu'il s'agit d'une invention bien orchestrée. Mais si contrefaçon de traces il y a eu, elle a été bien perpétrée: ce rituel de la porte est tissé de personnages, d'images, de couleurs qui font penser aux fables slaves ou balkaniques. Je pense alors aux terribles vérités du "Rameau d'or" que James Frazer a su dévoiler dans les fables: déicides, transferts de peine, boucs émissaires, le meurtre de l'esprit d'un arbre... Et si aujourd'hui on ne lit plus de fables, cela veut dire que la réalité les a dépassées et que le mal nous atteint directement dès notre enfance.

Voilà : ce spectacle est solaire comme un fable diurne, tout se déroule à la lumière du soleil, dans la réalité aveuglante du soleil de midi. En fait, un phare central illumine d'en haut un village de montagne, avec les portes comme des murs et les murs comme des portes: voilà que passe un chœur d'église mais d'une religion qui n'est pas la nôtre (mais quelle est notre religion?); voilà le bon voisinage de dames bavardes endimanchées dans leurs rubans colorés, aux chapeaux trop étroits et aux petits sacs de satin brandis comme une faux. Et voilà le démon niché dans l'horloge à coucou...

\section{Une fable morale, la morale de la fable}

Comme dans les autres spectacles de l'Odin, j'entrevois dans Kaosmos un ordre, mais pas un ordre linéaire: les actions se déroulent simultanément, selon une mise en scène qui semble chaotique et qui, en fait, est cosmique : et vraiment on ne pouvait 


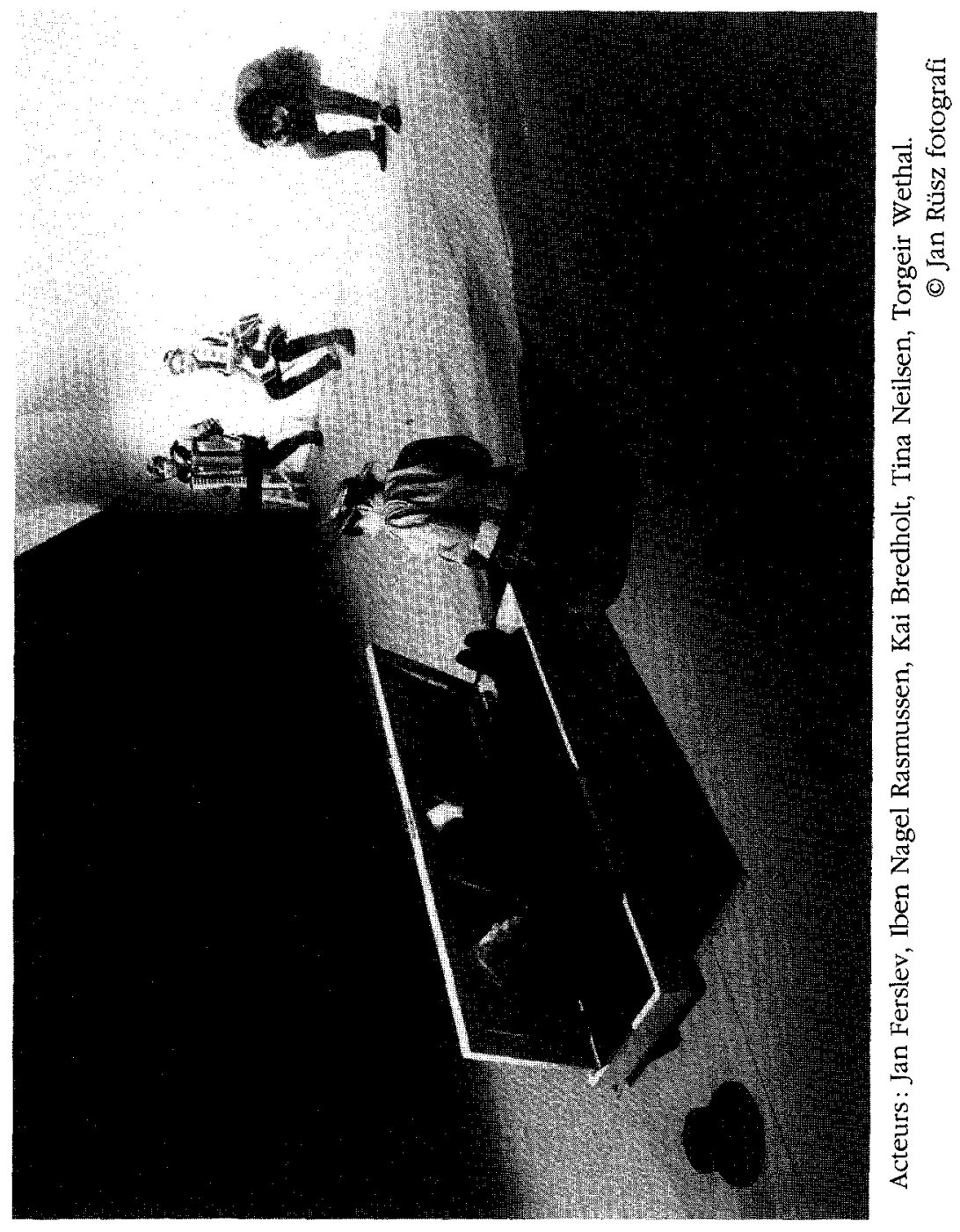


trouver meilleur titre, pour définir cette dramaturgie tourbillonnante (le désordre de l'univers) qui mêle les histoires, les langues, les personnages dans une fresque ouvrant de nombreuses perspectives. Cela a l'air d'une place publique et c'est, en fait, un voyage dans l'histoire.

L'histoire, en définitive, m'importe peu. Il y a d'ailleurs deux histoires et elles sont très simples. Dans la première un homme frappe à la porte pour entrer dans le règne de la félicité et du salut: un gardien le lui interdit et l'histoire dure toute une vie. Cette attente est la vie. La seconde histoire parle d'une mère qui cherche son enfant enlevé par la mort: cette recherche dure également toute une vie et cela lui permet de connaître tous les misères du monde. Aussi finalement quand elle retrouve son fils, la mère le préfère mort plutôt qu'en proie aux malheurs. Ces deux histoires - l'attente et la recherche - comme le dit candidement aussi le programme du spectacle, durent le temps d'une vie et deviennent "l'image de la fête et du gâchis de l'existence ".

On y verra une fête de village ou peut-être une représentation sacrée, une morality play mise en scène par la communauté dans laquelle ces deux histoires sont racontées en actions et en paroles. Les gens du pays participent en interprétant les différents personnages: une fois leurs parties respectives menées à terme, les gens se dépouillent des costumes des personnages qu'ils ont généreusement interprétés, sortent du spectacle et redeviennent le ramoneur, la laitière, l'étudiant, la maîtresse... peut-être en s'émerveillant que le voisin ou la voisine sache réciter aussi bien. J'habite un village et je sais reconnaitre les plaisirs innocents mais terriblement sérieux des gens de la campagne. Il y a la vieille dame, noble et élégante, pompeusement appelée Dame Musique, qui tisse inlassablement ses trames bavardes: la voix de Julia Varley est fine comme sa dentelle, elle coud l'histoire avec des "boucles-papillons" mais peut les faire devenir pierres de rivière. Il y a ensuite la mère qui cherche son fils enlevé par la mort: combien de sacrifices accomplit Roberta Carreri pour le ravoir, y compris même celui de s'arracher les yeux et de les jeter dans un lac. Tout est inutile, chère maman, quand tu auras passé ta vie à poursuivre cette entreprise et que tu auras atteint ton but, ton fils te répudiera.

Et il y a encore l'homme-qui-ne-veut-pas-mourir: on prendrait Torgeir Wethal pour l'idiot du village, le Saint Ingénu, 


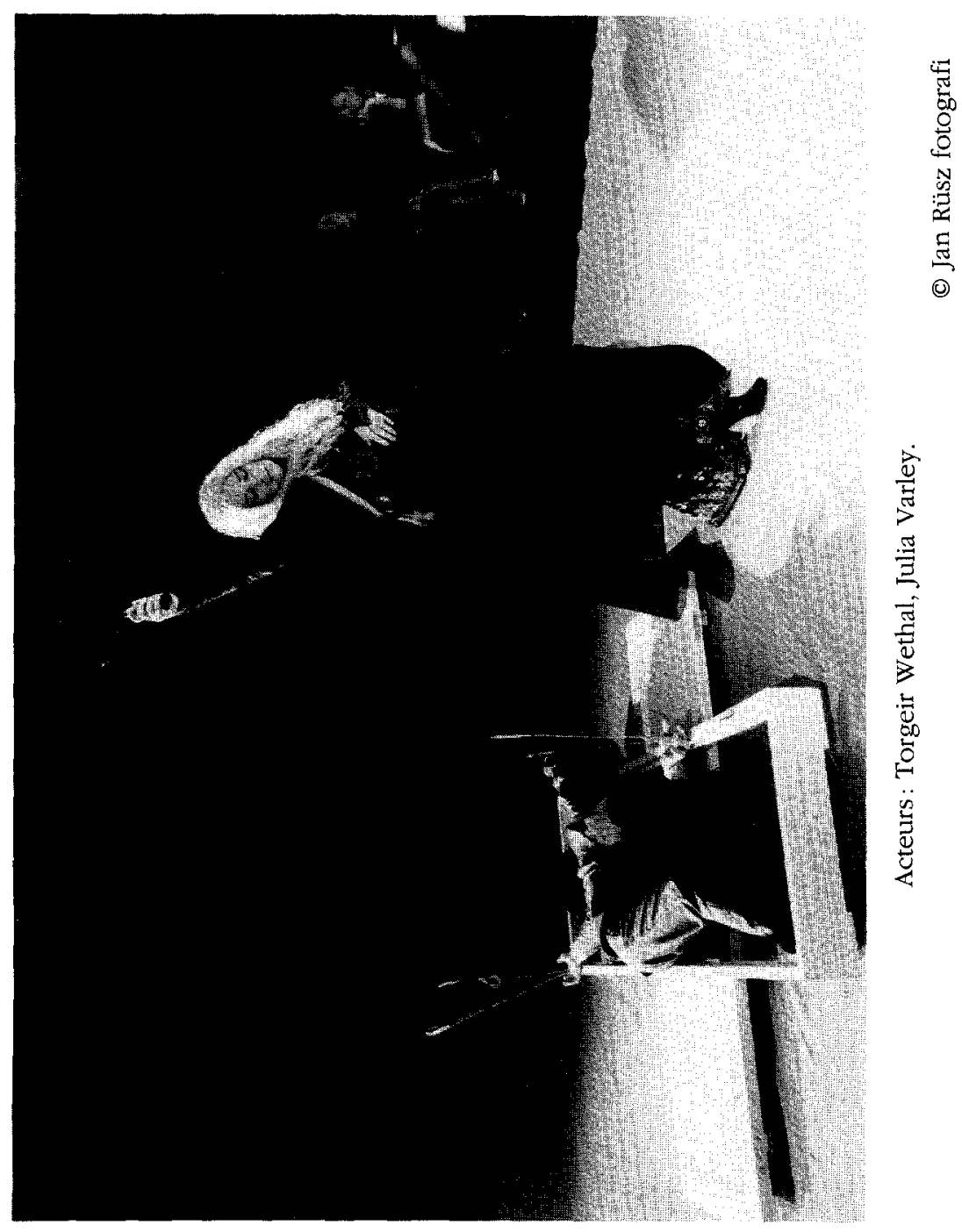


contraint de prendre pour femme, au dégoût général, celle qui fut la femme de tous - la diaphane Tina Nielsen, à la voix blonde comme ses chapeaux - et pourtant... Il y a enfin la maîtresse de campagne qui frappe à la porte toute sa vie: douce et persévérante Iben, elle mourra finalement sur le seuil où les noirs et joyeux gardiens - Jan Ferslev et Isabel Ubeda - chantent comme des anges. Et où un ange - le marin qui a vu la sirène - chante et joue comme l'endiablé Kai Bredholt.

Et puis il y a les objets dont le symbolisme magique fait sourire par l'efficace simplicité des trouvailles: comme ce livret inséré à l'intérieur d'un livre, provenant, à son tour, d'un livre plus grand, littéralement vissé à la porte. Chaque histoire, cher enfant, en contient une autre et de temps en temps un écrivain, un narrateur ou un acteur, pourquoi pas, les cloue toutes ensemble et les raconte. On a déjà parlé de la porte: les autres objets sont peu nombreux - un bracelet de pièces de monnaie, un morceau de tulle de mariée, les petits souliers d'argent d'un nouveau-né, les épis, la faux... - ils apparaissent comme des reliques d'un monde paysan, fabuleux et disparu. Ainsi il est même antédiluvien, ce voile déchiré qui, soudainement, se dresse sur la porte devenue barque, citation d'un manifeste du siècle passé: "Un spectre hante l'Europe" confesse le programme du spectacle.

Tout se déroule en fait dans ce village au cour de l'Europe de l'Est, dans les forêts montagneuses des Balkans: et par conséquent ce pourrait être tout sauf un drame moral. On suppose que le village existe réellement, quelque part en Transylvanie: mais le village se révèle: a) inventé de toutes pièces; b) mieux que vrai : vraisemblable. À chaque moment du spectacle on touche du doigt qu'une fable vraie supplante la réalité : avec Barba le pain quotidien de la fantaisie est plus crédible que l'ennuyeuse vérité. Je me souviens d'un épisode lié au drap qui, dans le film $L e c u i-$ rassé Potemkine, couvre les marins avant qu'ils soient fusillés sur le pont du navire. Quand on projeta le film, un vieil homme qui avait fait partie de l'équipage du cuirassé pendant la révolte de 1905, écrivit à Eisenstein: "Monsieur Eisenstein, je me suis vu dans votre film. J'étais l'un des marins sous le drap". Mais ce drap, Eisenstein l'avait inventé de toutes pièces: son génie s'était substitué à la mémoire de cet homme. J'ai souvent eu, à l'occasion des spectacles de l'Odin, la preuve de cette victoire de l'imagination sur les faits de l'histoire. 
66

Et les moteurs toujours en mouvement de cette imagination sont les acteurs (mais aucun n'est le protagoniste):

- Mais qui est le protagoniste de ce spectacle?

- Celui qui meurt à la fin.

- Est-ce que c'est ça le théâtre?

- Oui, c'est ça le théâtre: un fil fait de tours et d'astuces. Le personnage meurt et l'acteur revient à la vie.

Ainsi conversent l'homme-qui-ne-veut-pas-mourir et le gardien de la porte, en traversant la scène vers la fin du spectacle. Et nous n'avons pas de peine à les croire. S'il y a un homme-qui-plusque-les-autres-ressuscite, c'est l'acteur.

\section{Les limites de l'expérience}

Assister à un spectacle de l'Odin Teatret c'est faire une expérience. En parler n'est donc pas facile: même en revoyant plusieurs fois le spectacle dans le but de le saisir et de se l'approprier, ce n'est pas simple. S'il est dejà difficile, sinon impossible, de rendre une expérience par des mots, on rencontre dans les spectacles de l'Odin des conditions posant des limites à une description "objective". C'est pourquoi de prime abord: ce flux d'images, de réflexions, de mots que j'ai écrits, est une somme d'impressions et d'associations très personnelles : il s'agit de notes prises en marge du spectacle par un spectateur qui a été heureusement illusionné, c'est-à-dire ramené finalement "au jeu", mais ce sont des notes subjectives. Et donc: il est certain qu'on pourrait relever d'autres contenus dans le spectacle, comme il est sûr qu'un bon nombre des images que j'ai recueillies ne sont pas aussi univoques qu'elles me le sont apparues.

Certaines scènes peuvent, par exemple, se révéler à moi mais pas à un autre spectateur: le fait que les spectateurs soient assis divisés en deux groupes des deux côtés opposés de l'espace scénique favorise, littéralement, des visions opposées. Et encore: le fait que les acteurs jouent simultanément en plusieurs points de l'espace, implique que, même en restant attentif, on perd toujours une partie de ce qui se passe dans un autre endroit de la scène. En vérité, étant donné l'étendue modérée de l'espace, au lieu de perdre les actions qui ne sont pas directement regardées, on réussit à saisir quelque chose du coin de l'œeil, ce qui suffit pour com- 
prendre que quelque chose de différent s'y produit, mais ne permet pas de se rendre compte de ce qui s'est passé. Bref si l'on se concentre sur l'action des acteurs qui se tiennent devant soi, on perd les autres; si l'on allonge le regard vers les autres, on risque de perdre l'action au premier plan.

Et voici la sensation qui me désoriente le plus comme spectateur de l'Odin: ce n'est pas tant de ne pas comprendre la signification de ce qui est en train de se produire, mais plutôt le fait de réaliser qu'ailleurs, à peine un peu plus loin de l'endroit sur lequel j'ai les yeux fixés, quelque chose d'autre se passe, vient de se passer, mais que je l'ai aperçu trop tard. Je ne réussis plus à capter "ce qui s'est passé". Voici la sécurité dans laquelle, en tant que spectateur, je me sens menacé: non une simple incompréhension des contenus (qui sera jamais sûr de tout comprendre? de vouloir tout comprendre?) mais le fait de voir fuir la réalité et le temps sous ses yeux, et de ne pouvoir capter le changement, le devenir. Et néammoins je ne me sens pas pour autant comme faisant partie du public, comme un spectateur ordinaire: je me sens un spectateur encore plus curieux et motivé.

La vibrante présence scénique des acteurs, qui récitent à deux pas de soi avec une précision mathématique, les musiques et les chants qui les entortillent, les images fortes mais aux contenus ambigus, la vélocité de la représentation avec ses scènes simultanées dans l'entrelacement d'histoires au fond simples, le temps dense et suspendu dans un espace concentré, sont des données de fait plus que le style caractérisque non seulement de Kaosmos, mais de tous les spectacles originaux de l'Odin. Ce sont des spectacles pour les sens et pour l'âme. Mais ils semblent faits exprès pour discuter les règles du jeu.

Quelles sont les règles du jeu pour l'Odin? Comment parlet-on d'un spectacle de l'Odin? Et comment parle-t-on d'un tapis? Dans Kaosmos, on utilise un superbe tapis: les couleurs, les dessins et la brillance du poil révèlent, même à distance, une provenance précieuse et orientale. J'ai revu plusieurs fois le spectacle et ce tapis m'a suggéré l'idée que Kaosmos n'était pas une histoire dépeinte selon l'art figuratif. Kaosmos est un dessin d'art mais avec une logique différente: non un paysage traditionnel mais la décoration abstraite d'un tapis oriental.

Je vais peut-être dire une lapalissade, mais à l'origine, pour les tribus nomades de l'Asie centrale qui l'ont inventé, le tapis 
68

n'était pas une scénographie mais un outil nécessaire pour couvrir le sol sous la tente, et le transformer d'une fois à l'autre, en table, en couchette, lieu de rencontres douillettes et feutrées. Cet usage fonctionnel n'a pas empêché que le tapis soit décoré, mais les couleurs et les dessins sont totalement intégrés à la nature simple et pratique de l'objet. Un tapis est une surface horizontale qui doit être foulée, sur laquelle on peut s'asseoir et s'étendre, qui peut être touchée avec les mains: sa toison sera donc aussi souple que la fourrure de l'animal qu'il remplace et il suffira d'une simple division en espaces de couleur pour lui donner la vie tout comme une pure répétition de motifs symétriques est également suffisante pour rompre la monotonie de la couleur. Cette humilité est une conquête de l'art. En fait, si l'on accepte la règle d'or qui requiert de l'artiste qu'il utilise le minimum de moyens pour atteintre le maximum de résultat esthétique, un tapis petit et simple, venant d'une tribu nomade d'Anatolie est préférable aux tapis persans ornés d'arabesques infinies.

Aux grands tapis décoratifs des manufactures urbaines de Perse et de Turquie stylisant de mille manières les jardins et les eaux du paradis terrestre pour complaire aux riches demeures citadines, les tapis nécessaires des tribus nomades de l'Asie Centrale opposent peu de couleurs, en général le bleu et le rouge, et des dessins géométriques simples. Les tapis les plus parfaits n'ont pas de schémas compliqués et ne représentent rien de particulier: des dessins peu nombreux et essentiels reflètent le cosmos à leur niveau.

Si néammoins vous devez acheter un tapis, ne vous laissez pas séduire par les couleurs et les dessins, regardez-le bien à l'endroit et à l'envers et faites attention aux boucles: plus elles sont fines et serrées, et plus elles confèrent de valeur à la trame et à la durée.

\section{La leçon d'un tapis oriental}

Devant Kaosmos comme devant les autres spectacles de l'Odin, je ne me suis pas interrogé sur "le signifié". Non que ce ne fût pas une demande légitime mais parce que, au-delà de l'histoire ou des histoires représentées, au fond des thèmes choisis par l'Odin, on découvre toujours les grands leitmotive de l'existence: ainsi les doutes, les angoisses et les remords soulevés 
sont en effet universels, mais c'est justement pour cela qu'on ne peut les brandir; pour qu'ils aient une résonance chacun doit pudiquement les appréhender en soi-même, à sa façon. Ce qui, par contre, me surprend toujours, c'est qu'un spectacle théâtral, un jeu d'acteurs - même crédible - parviennent à me suggérer de tels contenus. C'est bien vrai: les spectacles parfaits, comme les tapis, sont une nécessité, ils n'ont pas de schémas compliqués et ne représentent rien de particulier. Des dessins essentiels, peu nombreux, reflètent le cosmos à leur niveau.

Pour le reste, de tous les nouds que les spectacles de l'Odin font émerger pour les théâtrologues, je n'en effleurerai qu'un, en raison de son caractère emblématique : celui du texte. Disons tout de suite que par " texte "j'entends les paroles que les acteurs prononcent durant le spectacle. Au cours des trente années d'histoire de l'Odin, Eugénio Barba a utilisé pour ses spectacles toute les formes possibles de texte - textes dramatiques à proprement parler, romans, contes, poésies, biographies, fables... - et il les a aussi exploités de toute les façons possibles: comme histoire de départ et comme histoire d'arrivée, comme écriture scénique ou comme base d'improvisations. Chaque spectacle est, en luimême, une histoire; il n'existe pas de règle fixe, si bien que dans certains spectacles on a utilisé toutes les formes de textes et toutes les manières possibles de les mettre en ouvre.

Ce qu'il est important de savoir à propos de ce travail avec les textes, c'est le fait que le collectif de théâtre - acteurs et metteur en scène - a réalisé, et réalise, le même processus de création qu'un écrivain, un peintre ou un artiste quel qu'il soit: le point de départ est souvent fortuit et banal, ce qui compte c'est la réélaboration. Et comme pour tout créateur, l'inventivité d'un groupe théâtral procède de la lutte entre hasard et rigueur, durant laquelle les transformations, les sauts qualitatifs, sont le résultat, non pas d'une intention ou d'un préjugé, mais d'un travail incessant sur la forme: travailler et retravailler sur la forme change aussi le contenu.

Il suffit de ne pas tomber dans les définitions-piège de "théâtre d'images", "théâtre de la parole" ou "théâtre de texte", pour se rendre compte qu'un spectacle de l'Odin est, techniquement parlant, une archive choisie, une dramaturgie a posteriori, un portrait distillé par le long travail préparatoire accompli par les acteurs et le metteur en scène - improvisations, compositions, répéti- 
70

tions - dont le poids spécifique est une masse d'énormes potentialités. Dans cette œuvre finale rigoureusement soignée dans chacune de ses parties comme un Nô japonais, le texte est seulement un des différents éléments utilisés, son rôle n'est ni premier, ni secondaire mais seulement un moyen bien étudié par l'acteur pour se révéler nécessaire au spectateur. Et pour cette raison nous sommes en présence, non pas d'un déluge de paroles mais d'une moisson de suggestions et de stimuli, d'un véritable art du gaspillage.

Comme dans une fête de la Renaissance italienne lorsque par la rencontre de gentilhommes et de gentes dames il naissait un temps suspendu, le temps de la fête, durant lequel on créait tableaux, musiques, poésies, danses, comédies... d'une œuvre se libèrent d'autres œuvres et ce qui compte le plus est la créativité, la fertilité des idées et la liberté de l'esprit.

\section{Pratiques de l'avenir}

Kaosmos est le plus récent spectacle de l'Odin Teatret. Cette année, le premier octobre, l'Odin parachève trente années d'activité: trente années de continuité et d'expérimentations, d'adieux et de retours, de spectacles et de livres, de bonnes et de mauvaises rencontres, de vies et d'amours autant qu'en peuvent concéder dix mille jours et dix mille nuits. Trente années vécues dans les lieux les plus divers du monde et à leurs antipodes: dans cette ville grise du Danemark qu'est Holstebro, où le groupe vit ses longues saisons de répétitions et sa quotidienneté fébrile. Les membres fondateurs du groupe sont au nombre de quatre: Eugenio Barba, Torgeir Wethal, Else Marie Laukvik et Iben Nagel Rasmussen. D'autres actrices très expérimentées comme Roberta Carreri et Julia Varley, se sont jointes à eux cela fait vingt ans. Par la suite les plus jeunes: Tina Nielsen, Isabel Ubeda, Kai Bredholt et surtout les deux musiciens Jan Ferslev et Frans Winther, dont le sound ${ }^{3}$ est devenu une dimension irremplaçable des derniers spectacles.

J'écrivais en 1974, au moment où j'ai connu l'Odin: "L'Odin est-il seulement un théâtre? Non, du moins pas selon les catégories habituelles. Un théâtre qui ne t'ennuie pas, qui ne te jette pas

3 En anglais dans le texte. 
au visage sa propagande, qui ne fréquente pas les circuits normaux; un théâtre qui t'absorbe en te retenant dans les plis de ton émotivité et qui va chercher son public parmi les étudiants, parmi les gens des villages, parmi les petits groupes de thêâtre qui travaillent au-delà des limites périphériques; un théâtre qui accepte de se nourrir mais dont les acteurs obtiennent un salaire à peine décent, et qui forme des acteurs de grand métier pour ensuite les voir s'en aller, loin du théâtre. Un théâtre comme celui-ci inflige un dur coup à la manie des étiquettes. Il renverse peut-être même l'acception traditionnelle d'un terme".

À vingt ans de distance, j'éprouve un double plaisir: de ne pas m'être trompé et de pouvoir aujourd'hui réécrire cela sans le "peut-être".

Traduction de Christine Borello, avec la collaboration de Ricardo Rosati 